\title{
Correlation Functions of Complex Matrix Models
}

\author{
M.C. Bergère \\ Service de Physique théorique, CEA-Saclay \\ F-91191 Gif sur Yvette, France \\ email: bergere@spht.saclay.cea.fr
}

October 29, 2005

\begin{abstract}
For a restricted class of potentials (harmonic+Gaussian potentials), we express the resolvent integral for the correlation functions of simple traces of powers of complex matrices of size $N$, in term of a determinant; this determinant is function of four kernels constructed from the orthogonal polynomials corresponding to the potential and from their Cauchy transform. The correlation functions are a sum of expressions attached to a set of fully packed oriented loops configurations; for rotational invariant systems, explicit expressions can be written for each configuration and more specifically for the Gaussian potential, we obtain the large $N$ expansion ('t Hooft expansion) and the so-called BMN limit.
\end{abstract}

\section{Introduction}

Given a potential $V\left(M, M^{+}\right)$where $M$ is a $N \times N$ matrix with complex elements, we define the partition function as

$$
Z=\int d M d M^{+} e^{-T r V\left(M, M^{+}\right)}
$$

where we integrate over all matrix elements. We define the correlation functions as

$$
<\prod_{i=1}^{n} O_{i}\left(M, M^{+}\right)>=\frac{1}{Z} \int d M d M^{+} \prod_{i=1}^{n} O_{i}\left(M, M^{+}\right) e^{-T r V\left(M, M^{+}\right)}
$$

Most observables $O\left(M, M^{+}\right)$can be expressed in terms of traces of product of the matrices $M$ and $M^{+}$; among these traces, we distinguish between "mixed traces" like $\operatorname{Tr}\left[M^{J_{1}}\left(M^{+}\right)^{K_{1}} M^{J_{2}}\left(M^{+}\right)^{K_{2}} \ldots\right]$ which are also called "words", 
and simple traces where there is only one type of matrix $\left(M\right.$ or $\left.M^{+}\right)$inside the trace. In this publication, we restrict ourselves to the case of simple traces

$$
<\prod_{i=1}^{p}\left[\operatorname{Tr} M^{J_{i}}\right] \prod_{i=1}^{q}\left[\operatorname{Tr}\left(M^{+}\right)^{K_{i}}\right]>\quad J_{i} \text { and } K_{i}>0
$$

for a restricted class of potentials (described below). We now explain the method used to obtain the above correlation functions.

If certain conditions on the potential $V\left(M, M^{+}\right)$are satisfied, we were able in Ref.[1] to calculate the following expectation values

$$
\frac{1}{Z} \int d M d M^{+} \frac{\prod_{i=1}^{L_{2}} \operatorname{det}\left(\eta_{i}^{*}-M^{+}\right)}{\prod_{i=1}^{M_{2}} \operatorname{det}\left(x_{i}^{*}-M^{+}\right)} \frac{\prod_{i=1}^{L_{1}} \operatorname{det}\left(\xi_{i}-M\right)}{\prod_{i=1}^{M_{1}} \operatorname{det}\left(y_{i}-M\right)} e^{-\operatorname{Tr} V\left(M, M^{+}\right)}
$$

for any numbers $L_{1}, M_{1}, L_{2}, M_{2}$ of determinants. The general result is essentially a determinant constructed from four kernels, each kernel is a function of the external sources $\xi_{i}, \eta_{i}^{*}, y_{i}, x_{i}^{*}$ which enter the orthogonal polynomials relative to the potential $V\left(z, z^{*}\right)$ and their Cauchy transform. The structure of this determinant is very dependant of the relative values of $L_{1}, M_{1}, L_{2}, M_{2}$. In this publication we are only interested in the special case where $L_{1}=M_{1}, L_{2}=M_{2}$ since in that case, if we perform the derivatives $\prod_{i=1}^{M_{2}}\left(\frac{\partial}{\partial \eta_{i}^{*}}\right)_{x_{i}^{*}=\eta_{i}^{*}} \prod_{i=1}^{M_{1}}\left(\frac{\partial}{\partial \xi_{i}}\right)_{y_{i}=\xi_{i}}$ on (1.4) we obtain

$$
\frac{1}{Z} \int d M d M^{+} \prod_{i=1}^{M_{2}} \operatorname{Tr} \frac{1}{\eta_{i}^{*}-M^{+}} \prod_{i=1}^{M_{1}} \operatorname{Tr} \frac{1}{\xi_{i}-M} e^{-\operatorname{Tr} V\left(M, M^{+}\right)}
$$

The above resolvent integrals are the generating functionals for the integrals

$$
\frac{1}{Z} \int d M d M^{+} \prod_{i=1}^{M_{2}} \operatorname{Tr}\left[\left(M^{+}\right)^{K_{i}}\right] \prod_{i=1}^{M_{1}} \operatorname{Tr}\left[(M)^{J_{i}}\right] e^{-\operatorname{Tr} V\left(M, M^{+}\right)}
$$

which describe the correlation functions (1.3).

Let us now describe the conditions required by the potential $V\left(M, M^{+}\right)$. Any matrix $M$ can be triangularized by a unitary matrix

$$
M=U[D+T] U^{+}
$$

where $D$ is the diagonal matrix of the eigenvalues $\left(z_{1}, \ldots, z_{N}\right)$ of $M$, and $T$ is an upper strictly triangular matrix. Consequently, any power of $M$ and $M^{+}$is of 
the form

$$
\begin{aligned}
M^{k} & =U\left[D^{k}+T_{k}\right] U^{+} \\
\left(M^{+}\right)^{k} & =U\left[\left(D^{+}\right)^{k}+T_{k}^{+}\right] U^{+}
\end{aligned}
$$

where $T_{k}$ is an upper triangular matrix which also depends of the eigenvalues $z_{i}$ for $k>1$. Clearly, we have

$$
\begin{gathered}
\operatorname{Tr} M^{k}=\sum_{i=1}^{N} z_{i}^{k} \\
\operatorname{Tr}\left(M^{+}\right)^{k}=\sum_{i=1}^{N}\left(z_{i}^{*}\right)^{k}
\end{gathered}
$$

On the other hand,

$$
\operatorname{Tr} M M^{+}=\sum_{i=1}^{N} z_{i} z_{i}^{*}+\operatorname{Tr} T T^{+}
$$

In the transformation (1.7),

$$
\begin{aligned}
\operatorname{det}\left(\xi_{i}-M\right) & =\operatorname{det}\left(\xi_{i}-D\right) \\
\operatorname{det}\left(\eta_{i}^{*}-M^{+}\right) & =\operatorname{det}\left(\eta_{i}^{*}-D^{+}\right)
\end{aligned}
$$

and the integration measure becomes

$$
d M d M^{+}=d U d T d T^{+} d D d D^{+} \prod_{i<j}\left|z_{i}-z_{j}\right|^{2}
$$

We note that $d M d M^{+}$represents $2 N^{2}$ variables of integration while $d U$ represents $N(N-1)$ variables of integration, $d T d T^{+}$represents $N(N-1)$ variables of integration and $d D d D^{+}$represents $2 N$ variables of integration. The Jacobian of the transformation is given by the product of two Vandermonde determinants $\Delta(z) \Delta\left(z^{*}\right)$ where

$$
\Delta(z)=\prod_{i<j}\left(z_{i}-z_{j}\right)=\left|\begin{array}{ccc}
\pi_{N-1}\left(z_{1}\right) & \ldots & \pi_{N-1}\left(z_{N}\right) \\
\ldots & \ldots & \ldots \\
\pi_{0}\left(z_{1}\right) & \ldots & \pi_{0}\left(z_{N}\right)
\end{array}\right|
$$

and where the polynomials $\left\{\pi_{0}(z), \pi_{1}(z), \pi_{2}(z), \ldots, \pi_{N-1}(z)\right\}$ are any set of monic polynomials of successive degree $0,1,2, \ldots, N-1$.

Consequently, if we choose the potential $V\left(M, M^{+}\right)$to be of the form

$$
V\left(M, M^{+}\right)=M M^{+}+V(M)+\bar{V}\left(M^{+}\right)
$$


we may separate the integrations over the unitary group $d U$ and over the upper triangular part $d T d T^{+} e^{-T r} T T^{+}$and these two contributions cancel in the ratio of the numerator and the denominator of (1.6). We are left with integrals over the eigenvalues only.

More generally, we consider the integrals $Z_{N}, I_{N}$ and $J_{N}$ and a potential $V\left(z, z^{*}\right)$ such that the following integrals exist

$$
\begin{gathered}
Z_{N}=\int d \mu\left(z, z^{*}\right) \\
I_{N}=\frac{1}{Z_{N}} \int d \mu\left(z, z^{*}\right) \prod_{j=1}^{N}\left\{\frac{\prod_{i=1}^{L_{2}}\left(z_{j}^{*}-\eta_{i}^{*}\right)}{\prod_{i=1}^{M_{2}}\left(z_{j}^{*}-x_{i}^{*}\right)} \frac{\prod_{i=1}^{L_{1}}\left(z_{j}-\xi_{i}\right)}{\prod_{i=1}^{M_{1}}\left(z_{j}-y_{i}\right)}\right\} \\
J_{N}=\frac{1}{Z_{N}} \int d \mu\left(z, z^{*}\right) \prod_{i=1}^{M_{2}}\left\{\sum_{j=1}^{N} \frac{1}{z_{j}^{*}-\eta_{i}^{*}}\right\} \prod_{i=1}^{M_{1}}\left\{\sum_{j=1}^{N} \frac{1}{z_{i}-\xi_{i}}\right\} \\
d \mu\left(z, z^{*}\right)=\prod_{i=1}^{N} d z_{i} d z_{i}^{*} \prod_{i<j}\left|z_{i}-z_{j}\right|^{2} e^{-\sum_{i=1}^{N} V\left(z_{i}, z_{i}^{*}\right)}
\end{gathered}
$$

The integrals $J_{N}$ are generating functionals for the integrals

$$
\frac{1}{Z_{N}} \int d \mu\left(z, z^{*}\right) \prod_{i=1}^{M_{2}}\left\{\sum_{j=1}^{N}\left(z_{j}^{*}\right)^{K_{i}}\right\} \prod_{i=1}^{M_{1}}\left\{\sum_{j=1}^{N}\left(z_{j}\right)^{J_{i}}\right\}
$$

A second condition on $V\left(M, M^{+}\right)$is that $V\left(z, z^{*}\right)$ is real and admits a set of orthogonal polynomials so that the technique of Ref[1] can be applied (the reality of $V\left(z, z^{*}\right)$ could be forgotten at the price of introducing biorthogonal polynomials but we shall not consider this case). We introduce the infinite set $\left\{p_{n}(z)\right\}$ of orthogonal, monic polynomials such that

$$
\int d^{2} z p_{m}^{*}(z) p_{n}(z) e^{-V\left(z, z^{*}\right)}=h_{n} \delta_{n m}
$$

where $p_{m}^{*}(z)$ is a short notation for $\left[p_{m}(z)\right]^{*}$. Then, we consider the measure

$$
d \mu\left(z, z^{*} ; \xi_{i}, \eta_{i}^{*} ; y_{i}, x_{i}^{*}\right)=d^{2} z \frac{\prod_{i=1}^{L_{2}}\left(z^{*}-\eta_{i}^{*}\right)}{\prod_{i=1}^{M_{2}}\left(z^{*}-x_{i}^{*}\right)} \frac{\prod_{i=1}^{L_{1}}\left(z-\xi_{i}\right)}{\prod_{i=1}^{M_{1}}\left(z-y_{i}\right)} e^{-V\left(z, z^{*}\right)}
$$


We proved in Ref.[1] the existence and we constructed explicitely the set of monic, biorthogonal polynomials $q_{n}\left(z ; \xi_{i}, \eta_{i}^{*} ; y_{i}, x_{i}^{*}\right)$ and $q_{n}^{*}\left(z ; \eta_{i}, \xi_{i}^{*} ; x_{i}, y_{i}^{*}\right)$ satisfying

$$
\int d \mu\left(z, z^{*} ; \xi_{i}, \eta_{i}^{*} ; y_{i}, x_{i}^{*}\right) q_{m}^{*}\left(z ; \eta_{i}, \xi_{i}^{*} ; x_{i}, y_{i}^{*}\right) \quad q_{n}\left(z ; \xi_{i}, \eta_{i}^{*} ; y_{i}, x_{i}^{*}\right)=\left\|q_{n}\right\|^{2} \quad \delta_{n m}
$$

This construction is a generalization of Christoffel's result [2] which shows that given a positive Borel measure of one variable $d \mu(x)$ on the real line and its infinite set of orthogonal polynomials, it is possible to construct an infinite set of orthogonal polynomials for the measure

$$
d \mu\left(x ; \xi_{i}\right)=d \mu(x) \prod_{i=1}^{L}\left(x-\xi_{i}\right)
$$

This result was extended to measures with external sources at the denominator by Uvarov [3] in 1969 and recently by Fyodorov and Strahov [4]. In 2003, Akemann and Vernizzi extended Christoffel's result to measures on the complex plane with external sources at the numerator [5] (see also Ref.[6]); their work was generalized to sources at the denominator as well in Ref.[1] and [7].

The consequence of the existence of biorthogonal polynomials for the measure (1.18) is that

$$
\begin{aligned}
Z_{N} & =N ! \prod_{i=0}^{N-1} h_{i} \\
Z_{N} \quad I_{N} & =N ! \prod_{i=0}^{N-1}\left\|q_{i}\right\|^{2}
\end{aligned}
$$

In the case $L_{1}=M_{1}, L_{2}=M_{2}$, the pseudonorms $\left\|q_{i}\right\|^{2}$ are found in Ref.[1] to be the ratio of two determinants

$$
\left\|q_{i}\right\|^{2}=h_{i} \frac{D_{i}}{D_{i-1}} \quad i \geq 0
$$

where $D_{n}$ is the determinant

$$
\left|\begin{array}{cccccc}
N_{n}\left(\xi_{1}, y_{1}\right) & \ldots & N_{n}\left(\xi_{M_{1}}, y_{1}\right) & A_{n}\left(x_{1}^{*}, y_{1}\right) & \ldots & A_{n}\left(x_{M_{2}}^{*}, y_{1}\right) \\
\ldots & \ldots & \ldots & \ldots & \ldots & \ldots \\
N_{n}\left(\xi_{1}, y_{M_{1}}\right) & \ldots & N_{n}\left(\xi_{M_{1}}, y_{M_{1}}\right) & A_{n}\left(x_{1}^{*}, y_{M_{1}}\right) & \ldots & A_{n}\left(x_{M_{2}}^{*}, y_{M_{1}}\right) \\
K_{n}\left(\xi_{1}, \eta_{1}^{*}\right) & \ldots & K_{n}\left(\xi_{M_{1}}, \eta_{1}^{*}\right) & N_{n}^{*}\left(\eta_{1}, x_{1}\right) & \ldots & N_{n}^{*}\left(\eta_{1}, x_{M_{2}}\right) \\
\ldots & \ldots & \ldots & \ldots & \ldots & \ldots \\
K_{n}\left(\xi_{1}, \eta_{M_{2}}^{*}\right) & \ldots & K_{n}\left(\xi_{M_{1}}, \eta_{M_{2}}^{*}\right) & N_{n}^{*}\left(\eta_{M_{2}}, x_{1}\right) & \ldots & N_{n}^{*}\left(\eta_{M_{2}}, x_{M_{2}}\right)
\end{array}\right|
$$


and $D_{-1}$ is defined in (1.32). Consequently, in the case $L_{1}=M_{1}, L_{2}=M_{2}$ the integral $I_{N}$ is found to be

$$
I_{N}=(-)^{\frac{M_{1}\left(M_{1}-1\right)}{2}}(-)^{\frac{M_{2}\left(M_{2}-1\right)}{2}} \frac{\prod_{i, j=1}^{M_{2}}\left(x_{i}^{*}-\eta_{j}^{*}\right) \prod_{i, j=1}^{M_{1}}\left(y_{i}-\xi_{j}\right)}{\Delta\left(x^{*}\right) \Delta(y) \Delta\left(\eta^{*}\right) \Delta(\xi)} D_{N-1}
$$

Then, by application of the derivatives $\prod_{i=1}^{M_{2}}\left(\frac{\partial}{\partial \eta_{i}^{*}}\right)_{x_{i}^{*}=\eta_{i}^{*}} \prod_{i=1}^{M_{1}}\left(\frac{\partial}{\partial \xi_{i}}\right)_{y_{i}=\xi_{i}}$ on (1.24), we proved in Ref.[1] that

$$
J_{N}=" D_{N-1} "
$$

where " $D_{n}$ " is the "subtracted determinant"

$$
\left|\begin{array}{cccccc}
H_{n}\left(\xi_{1}, \xi_{1}\right) & \ldots & N_{n}\left(\xi_{M_{1}}, \xi_{1}\right) & A_{n}\left(\eta_{1}^{*}, \xi_{1}\right) & \ldots & A_{n}\left(\eta_{M_{2}}^{*}, \xi_{1}\right) \\
\ldots & \ldots & \ldots & \ldots & \ldots & \ldots \\
N_{n}\left(\xi_{1}, \xi_{M_{1}}\right) & \ldots & H_{n}\left(\xi_{M_{1}}, \xi_{M_{1}}\right) & A_{n}\left(\eta_{1}^{*}, \xi_{M_{1}}\right) & \ldots & A_{n}\left(\eta_{M_{2}}^{*}, \xi_{M_{1}}\right) \\
K_{n}\left(\xi_{1}, \eta_{1}^{*}\right) & \ldots & K_{n}\left(\xi_{M_{1}}, \eta_{1}^{*}\right) & H_{n}^{*}\left(\eta_{1}, \eta_{1}\right) & \ldots & N_{n}^{*}\left(\eta_{M_{2}}, \eta_{1}\right) \\
\ldots & \ldots & \ldots & \ldots & \ldots & \ldots \\
K_{n}\left(\xi_{1}, \eta_{M_{2}}^{*}\right) & \ldots & K_{n}\left(\xi_{M_{1}}, \eta_{M_{2}}^{*}\right) & N_{n}^{*}\left(\eta_{1}, \eta_{M_{2}}\right) & \ldots & H_{n}^{*}\left(\eta_{M_{2}}, \eta_{M_{2}}\right)
\end{array}\right|
$$

The determinant $D_{n}$ is expressed in terms of four kernels $K_{n}, N_{n}, N_{n}^{*}, A_{n}$ which are defined with some of their properties at the end of this introduction. The determinant " $D_{n}$ " is obtained from the determinant $D_{n}$ by changing all kernels $N_{n}$ on the diagonal into $H_{n}$, changing all $y_{k}$ into $\xi_{k}$, all $x_{k}^{*}$ into $\eta_{k}^{*}$ and finally by ignoring all double poles at $\xi_{j}=\xi_{k}$ and at $\eta_{j}^{*}=\eta_{k}^{*}$ (this operation is denoted by " ") as we develop the determinant (1.26) with terms like $N_{n}\left(\xi_{j}, \xi_{k}\right) N_{n}\left(\xi_{k}, \xi_{j}\right)$ or $N_{n}^{*}\left(\eta_{k}, \eta_{j}\right) N_{n}^{*}\left(\eta_{j}, \eta_{k}\right)$. In fact, from (1.30.b) we observe that there is no single poles either at $\xi_{j}=\xi_{k}$ or at $\eta_{j}^{*}=\eta_{k}^{*}$ since the residues are zero

$$
" N_{n}\left(\xi_{j}, \xi_{k}\right) N_{n}\left(\xi_{k}, \xi_{j}\right) "=H_{n}\left(\xi_{j}, \xi_{k}\right) H_{n}\left(\xi_{k}, \xi_{j}\right)+\frac{H_{n}\left(\xi_{j}, \xi_{k}\right)-H_{n}\left(\xi_{k}, \xi_{j}\right)}{\xi_{j}-\xi_{k}}
$$

In section 2 , we develop the determinant " $D_{n}$ " in terms of fully packed oriented loops configurations. Then, in section 3, we give an expression for each configuration in the case of rotationnally invariant systems ; finally in section 4, we use the Gaussian potential to obtain the correlation functions (1.3) which in that case are nothing but the number of graphs obtained from (1.6) by Wick's contraction. The large $N$ expansion (where $N$ is the size of the matrix) is considered and provides the number of graphs according to their genus ( $t$ 'Hooft expansion [8]); another limit is also considered, namely the so-called BMN limit [9], where $J_{i}, K_{i}$ in (1.3) and $N$ are large but the ratios $\frac{J_{i}}{\sqrt{N}}$ and $\frac{K_{i}}{\sqrt{N}}$ are constant. Similar results were presented in [10] and [11]. 
The end of this introduction is devoted to the description of the kernels $K_{n}, N_{n}, A_{n}$ and to some of their properties. We refer to Ref.[1] for the technical details; here, we simply give the definition of these kernels

$$
K_{n}\left(\xi, \eta^{*}\right)=\sum_{i=0}^{n} \frac{p_{i}(\xi) p_{i}^{*}(\eta)}{h_{i}}
$$

We introduce the following integrals (in the case of potentials of one variable, the integral (1.29.a) is called the Cauchy-Hilbert transform of the corresponding polynomial)

$$
\begin{aligned}
t_{n}(y) & =\int d^{2} z p_{n}^{*}(z) \frac{1}{z-y} e^{-V\left(z, z^{*}\right)} \\
Q\left(x^{*}, y\right) & =\int d^{2} z \frac{1}{\left(z^{*}-x^{*}\right)(z-y)} e^{-V\left(z, z^{*}\right)}
\end{aligned}
$$

then,

$$
\begin{aligned}
H_{n}(\xi, y) & =\sum_{i=0}^{n} \frac{p_{i}(\xi) t_{i}(y)}{h_{i}} \\
N_{n}(\xi, y) & =\frac{1}{y-\xi}+H_{n}(\xi, y) \\
A_{n}\left(x^{*}, y\right) & =\sum_{i=0}^{n} \frac{t_{i}^{*}(x) t_{i}(y)}{h_{i}}-Q\left(x^{*}, y\right)
\end{aligned}
$$

In (1.22), we also use the extensions

$$
\begin{aligned}
K_{-1}\left(\xi, \eta^{*}\right) & =0 \\
N_{-1}(\xi, y) & =\frac{1}{y-\xi} \\
A_{-1}\left(x^{*}, y\right) & =-Q\left(x^{*}, y\right)
\end{aligned}
$$

and the determinant

$$
\begin{aligned}
& D_{-1}=\left|\begin{array}{cc}
N_{-1}\left(\xi_{j}, y_{i}\right) & A_{-1}\left(x_{l}^{*}, y_{i}\right) \\
0 & N_{-1}^{*}\left(\eta_{k}, x_{l}\right)
\end{array}\right| \\
& D_{-1}=\left|N_{-1}\left(\xi_{j}, y_{i}\right)\right| \cdot\left|N_{-1}^{*}\left(\eta_{k}, x_{l}\right)\right|
\end{aligned}
$$

where

$$
\begin{aligned}
& \left|N_{-1}\left(\xi_{j}, y_{i}\right)\right|=\left|\begin{array}{ccc}
\frac{1}{y_{1}-\xi_{1}} & \ldots & \frac{1}{y_{1}-\xi_{M_{1}}} \\
\ldots & \ldots & \ldots \\
\frac{1}{y_{M_{1}}-\xi_{1}} & \ldots & \frac{1}{y_{M_{1}}-\xi_{M_{1}}}
\end{array}\right| \\
& \left|N_{-1}\left(\xi_{j}, y_{i}\right)\right|=(-)^{\frac{M_{1}\left(M_{1}-1\right)}{2}} \frac{\Delta(y) \Delta(\xi)}{\prod_{i, j}\left(y_{i}-\xi_{j}\right)}
\end{aligned}
$$


In Ref.[1], we prove the following properties

$$
\begin{aligned}
& \sum_{i=0}^{\infty} \frac{p_{i}(\xi) t_{i}(y)}{h_{i}}=\frac{1}{\xi-y} \\
& \sum_{i=0}^{\infty} \frac{t_{i}^{*}(x) t_{i}(y)}{h_{i}}=Q\left(x^{*}, y\right)
\end{aligned}
$$

so that we have the formal power series

$$
\begin{gathered}
N_{n}(\xi, y)=-\sum_{i=n+1}^{\infty} \frac{p_{i}(\xi) t_{i}(y)}{h_{i}} \\
A_{n}\left(x^{*}, y\right)=-\sum_{i=n+1}^{\infty} \frac{t_{i}^{*}(x) t_{i}(y)}{h_{i}}
\end{gathered}
$$

Finally, we have the asymptotic behaviour

$$
\begin{aligned}
t_{n}(y) & \sim-\frac{h_{n}}{y^{n+1}} \text { as } y \rightarrow \infty \\
N_{n}(\xi, y) & \sim \frac{p_{n+1}(\xi)}{y^{n+2}} \text { as } y \rightarrow \infty \\
A_{n}\left(x^{*}, y\right) & \sim \frac{t_{n+1}^{*}(x)}{y^{n+2}} \text { as } y \rightarrow \infty
\end{aligned}
$$

\section{The graph description of the determinant " $D_{n}$ "}

The determinant " $D_{n}$ " as given in (1.26) is a sum of products of oriented propagators joining the various points (for simplicity, we label the points and the corresponding variables by the same letter). Let us split the plane into two parts separated by a border line; on the right part we draw the various points $\eta_{i}^{*}$ and in the left part we draw the various points $\xi_{i}$. As we develop the determinant, every time we meet a kernel $A_{n}\left(\eta_{i}^{*}, \xi_{j}\right)$ we draw an oriented propagator from the point $\eta_{i}^{*}$ towards the point $\xi_{j}$ crossing the border from right to left; similarly with the kernel $K_{n}\left(\xi_{i}, \eta_{j}^{*}\right)$ we draw an oriented propagator from the point $\xi_{i}$ towards the point $\eta_{j}^{*}$ crossing the border from left to right. Then, inside the $\xi$-region we draw an oriented propagator from the point $\xi_{i}$ towards the point $\xi_{j}$ corresponding to the kernel $N_{n}\left(\xi_{i}, \xi_{j}\right)$; in addition, the diagonal part of " $D_{n}$ " defines self closing loops on one point $\xi_{i}$ from the kernel $H_{n}\left(\xi_{i}, \xi_{i}\right)$. Similarly, inside the $\eta^{*}$-region we draw an oriented propagator from the point $\eta_{i}^{*}$ towards the point $\eta_{j}^{*}$ corresponding to the kernel $N_{n}^{*}\left(\eta_{j}, \eta_{i}\right)$; in addition, the diagonal part of " $D_{n}$ " defines self closing loops on one point $\eta_{i}^{*}$ from the kernel $H_{n}^{*}\left(\eta_{i}, \eta_{i}\right)$. 
As we develop the determinant, the product of propagators describes a number of disjoint oriented closed loops $\gamma_{a}$ (including self-loop with one point) so that any point $\xi_{i}$ or $\eta_{i}^{*}$ belongs to one closed loop $\gamma_{a}$ and only one; we call such a configuration "fully packed oriented loops configuration" which we denote by $\varpi$ (fig.1); the number of oriented loops in $\varpi$ is denoted \#( $\#)$

$$
\varpi=\cup_{a=1}^{\#(\varpi)} \gamma_{a}
$$
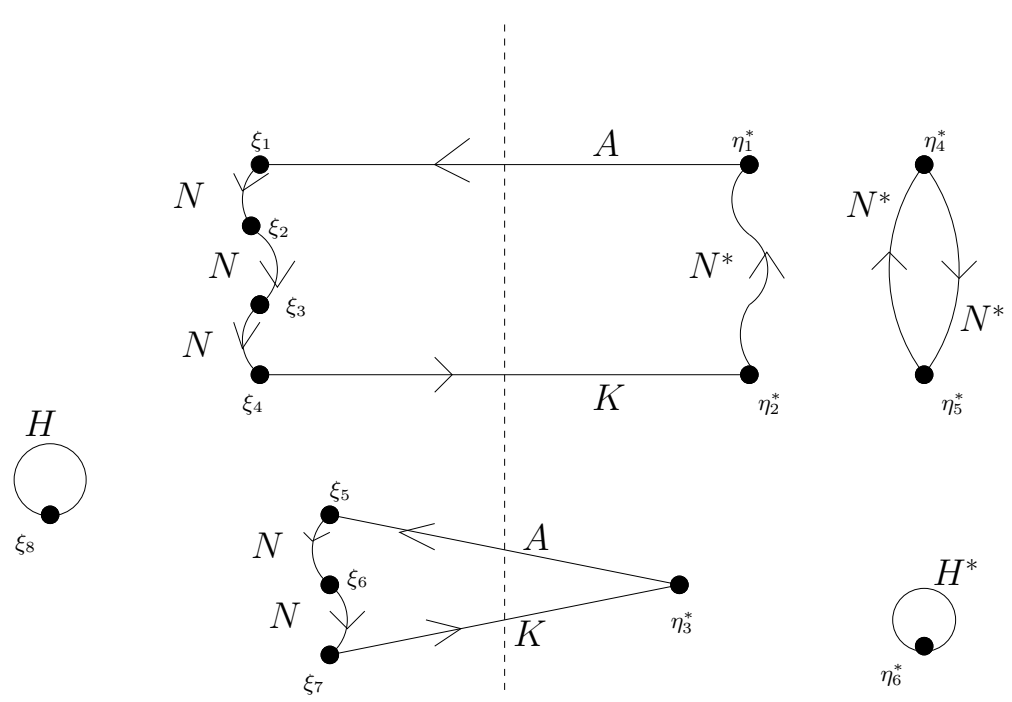

fig.1 : contribution to $W_{8,6}\left(\xi_{1}, \ldots, \xi_{8} ; \eta_{1}^{\star}, \ldots, \eta_{6}^{\star}\right)$

The determinant " $D_{n}$ " is the sum over the $\left(M_{1}+M_{2}\right)$ ! configurations $\varpi$

$$
" D_{n} "=(-)^{M_{1}+M_{2}} \sum_{\varpi} \prod_{a=1}^{\#(\varpi)}\left(-I_{\gamma_{a}}\right)
$$

Given an oriented loop $\gamma$, which is not made of $N$ propagators alone and/or of $N^{*}$ propagators alone, the amplitude $I_{\gamma}$ is the product of a certain number $q>0$ of propagators $K_{n}$ and of the same number $q$ of propagators $A_{n}$; the remaining propagators describe $q$ chains of propagators $N$ and $q$ chains of propagators $N^{*}$ (such chains may be reduced to a single point). We call skeleton graph an oriented loop $\gamma$ without $N$ and $N^{*}$ propagators. To any oriented loop $\gamma$ we associate a skeleton graph by contraction of each of its chains of propagators $N$ or $N^{*}$ into a point.

Along a given chain, the propagators $N$ or $N^{*}$ have poles when the $\xi^{\prime} s$ or the $\eta^{\prime} s$ coincide. However, these poles cancel when we sum over all permutations of the $\xi^{\prime} s$ or of the $\eta^{\prime} s$ inside a given chain of propagators $N$ or $N^{*}$. In appendix 
A we show how the poles cancel when we sum over several configurations which have the same associated skeleton graph.

For a given potential $V\left(z, z^{*}\right)$, we must calculate the orthogonal polynomials $p_{n}(z)$ and their Cauchy transform $t_{n}(z)$ in order to evaluate the kernels which are the propagators of the graphs. Of course, each case is a specific case; however, some general structure can be obtained in the special case where the system (potential and domain of integration) is rotationnally invariant.

\section{The $\frac{1}{\xi}, \frac{1}{\eta^{*}}$ expansion in the case of the rota- tionnally invariant systems}

The expressions for the four kernels $K_{n}, N_{n}, N_{n}^{*}, A_{n}$ simplify greatly in the case of rotationnally invariant systems because the angular integrations in (1.17) is trivially performed. Given a potential $V\left(z z^{*}\right)$ and a disc of radius $R$ which might be the complex plane $(R=\infty)$ if $\exp \left(-V\left(z z^{*}\right)\right)$ decreases strongly enough at $\infty$. In that case, the orthogonal polynomials are the so-called Ginibre's polynomials

$$
p_{n}(z)=z^{n}
$$

and their norms squared are

$$
h_{n}=2 \pi \int_{0}^{R} d \rho \rho^{2 n+1} e^{-V\left(\rho^{2}\right)}
$$

In the Gaussian case where $V\left(z z^{*}\right)=z z^{*}$ and $R=\infty$,

$$
h_{n}=\pi \Gamma(n+1)
$$

The function $t_{n}(y)$ defined in (1.29.a) is found to be

$$
t_{n}(y)=-\frac{h_{n}}{y^{n+1}}, \quad|y|>R
$$

if $R=\infty$ this result is still valid up to exponentially small terms when $y \rightarrow \infty$ and can be used as a formal power series (see also (1.34.a)).

According to (1.28) and (1.30) the formal power series for the kernels $K_{n}, H_{n}, N_{n}$ and $A_{n}$ are

$$
\begin{aligned}
& K_{n}\left(\xi, \eta^{*}\right)=\sum_{j=0}^{n} \frac{\left(\xi \eta^{*}\right)^{j}}{h_{j}} \\
& H_{n}\left(\xi_{i}, \xi_{j}\right)=-\frac{1}{\xi_{j}^{n+1}} \frac{\xi_{i}^{n+1}-\xi_{j}^{n+1}}{\xi_{i}-\xi_{j}} \quad \xi_{i} \neq \xi_{j}
\end{aligned}
$$


so that

$$
\begin{aligned}
H_{n}\left(\xi_{i}, \xi_{i}\right) & =-\frac{n+1}{\xi_{i}} \\
N_{n}\left(\xi_{i}, \xi_{j}\right) & =\left(\frac{\xi_{i}}{\xi_{j}}\right)^{n+1} \frac{1}{\xi_{j}-\xi_{i}}
\end{aligned}
$$

Finally,

$$
A_{n}\left(\eta^{*}, \xi\right)=-\sum_{i=n+1}^{\infty} \frac{h_{i}}{\left(\xi \eta^{*}\right)^{i+1}}
$$

It is now possible to calculate for any closed loop $\gamma$ the corresponding amplitude $I_{\gamma}$ which contributes to the value of the determinant " $D_{n}$ ".

$\left.1^{\circ}\right)$ the $\frac{1}{\xi}, \frac{1}{\eta^{*}}$ expansion for the skeleton graphs: given a skeleton graph with $q>0$ propagators $A$ and $q$ propagators $K$, the expression for $I_{\gamma}$ can be written

$$
I_{\gamma}=\prod_{k=1}^{q} A_{n}\left(\eta_{\alpha_{k}}^{*}, \xi_{\alpha_{k}}\right) \prod_{i}^{q} K_{n}\left(\xi_{\alpha_{k}}, \eta_{\alpha_{k+1}}^{*}\right) \quad \text {, with } \quad \eta_{\alpha_{q+1}}^{*}=\eta_{\alpha_{1}}^{*}
$$

As we expand the propagators $A_{n}$ and $K_{n}$ according to (3.5.a) and (3.7) we introduce an internal momentum $i_{k}$ for $A_{n}\left(\eta_{\alpha_{k}}^{*}, \xi_{\alpha_{k}}\right)$ and an internal momentum $j_{k}$ for $K_{n}\left(\xi_{\alpha_{k}}, \eta_{\alpha_{k+1}}^{*}\right)$; the summation over $i_{k}$ runs from $n+1$ to $\infty$ while the summation over $j_{k}$ runs from 0 to $n$. By convention, we extend the definition of $h_{j}$ to negative $j^{\prime} s$ as $h_{j<0}=\infty$ so that in $K_{n}$ we may eventually ignore the lower limit of summation.

The expansion of $I_{\gamma}$ is of the form

$$
I_{\gamma}=\sum_{\left\{J^{\prime} s>0, K^{\prime} s>0\right\}} \prod_{k=1}^{q}\left(\frac{1}{\xi_{\alpha_{k}}^{J_{k}+1}}\right) \prod_{k=1}^{q}\left(\frac{1}{\eta_{\alpha_{k}}^{K_{k}+1}}\right)^{*} \quad I\left(J_{k}, K_{k}\right)
$$

where

$$
I\left(J_{k}, K_{k}\right)=(-)^{q} \sum_{D_{\left\{J_{k}, K_{k}\right\}}} \frac{h_{i_{1}} \ldots h_{i_{q}}}{h_{j_{1}} \ldots h_{j q}}
$$

and $D_{\left\{J_{k}, K_{k}\right\}}$ is the summation domain for the indices $i_{k}$ and $j_{k}$. The determination of this domain is the main difficulty. Clearly, all the indices $i^{\prime} s$ and $j^{\prime} s$ are fixed in terms of the $J^{\prime} s$ and the $K^{\prime} s$ up to a translation.

We first describe some simple exemples:

a) let us consider the graph $\gamma_{1}$ (fig.2). 


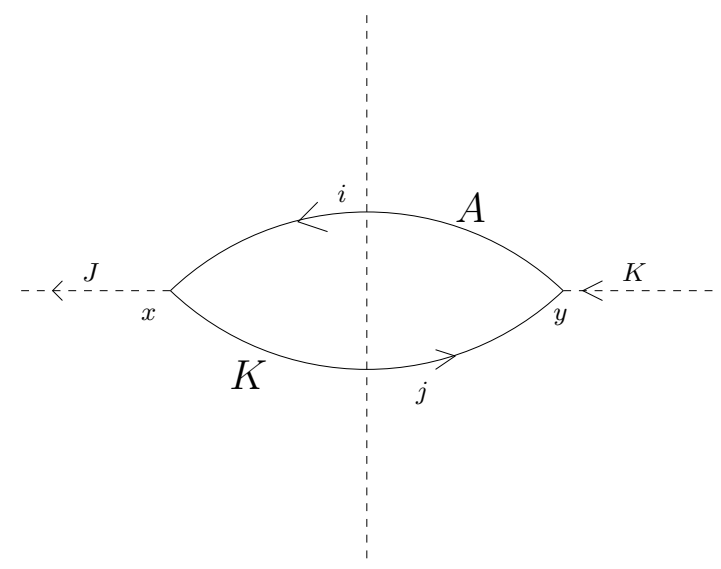

fig. 2: graph $\gamma_{1} \rightarrow W_{1,1}^{c}(x, y)$

In that case, the momentum conservation law is

$$
i-j=J=K
$$

so that the domain of summation for the momentum $j$ is

$$
n-J+1 \leq j \leq n
$$

Consequently,

$$
I_{\gamma_{1}}(J, K)=-\delta_{J, K} \sum_{j=n-J+1}^{n} \frac{h_{j+J}}{h_{j}}
$$

The equation (3.13) is still valid in the case where $n-J+1<0$ with the convention $h_{j<0}=\infty$. We define the characteristic function for the skeleton graph $\gamma_{1}$

$$
\Phi_{\gamma_{1}}(n, J)=\sum_{j=0}^{n} \frac{h_{j+J}}{h_{j}}
$$

then,

$$
I_{\gamma_{1}}(J, K)=-\delta_{J, K} \quad\left(1-T_{J}\right) \Phi_{\gamma_{1}}(n, J)
$$

where the operator $T_{J}$ is a translation operator over $n$

$$
T_{J} f(n)=f(n-J)
$$


b) let us consider the graph $\gamma_{2}$ (fig.3).

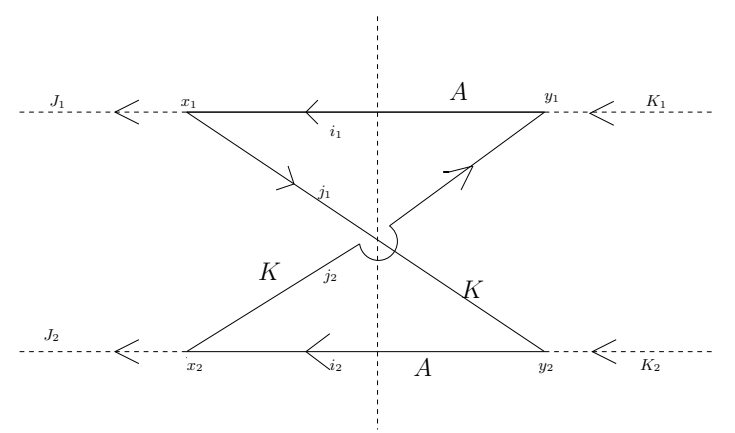

fig. 3: graph $\gamma_{2} \rightarrow$ contribution to $W_{2,2}^{c}\left(x_{1}, x_{2} ; y_{1}, y_{2}\right)$

Choosing $j_{2}=j$ as the independant loop momentum, we observe the following constraints

$$
\begin{aligned}
& j \geq n+1-K_{1}, \quad j \geq n+1-J_{2} \\
& j \leq n-K_{1}+J_{1}, \quad j \leq n \\
& J=J_{1}+J_{2}=K_{1}+K_{2}=K
\end{aligned}
$$

Consequently, we obtain

$$
\begin{aligned}
I_{\gamma_{2}}\left(J_{1}, J_{2}, K_{1}, K_{2}\right) & =\delta_{J, K} O\left(J_{i}, K_{i}\right) \Phi_{\gamma_{2}}\left(n, J_{i}, K_{i}\right) \\
O\left(J_{i}, K_{i}\right) & =T_{\operatorname{Sup}\left(K_{1}-J_{1}, 0\right)}-T_{I n f\left(K_{1}, J_{2}\right)} \\
\Phi_{\gamma_{2}}\left(n, J_{i}, K_{i}\right) & =\sum_{j=0}^{n} \frac{h_{j+K_{1}} \quad h_{j+J_{2}}}{h_{j+K_{1}-J_{1}} h_{j}}
\end{aligned}
$$

c) the generalization to the skeleton graph $\gamma_{q}$ with $q$ pairs of propagators $A_{n}$ and $K_{n}$ is now straightforward. We define

$$
J=\sum_{k=1}^{q} J_{k} \quad K=\sum_{k=1}^{q} K_{k}
$$

and we choose $j=j_{q}$ as independant loop momentum so that

$$
i_{k}=j_{q}+b_{k} \quad j_{k}=j_{q}+a_{k}
$$

with

$$
\begin{aligned}
a_{k} & =\sum_{p=1}^{k}\left(K_{p}-J_{p}\right), \quad a_{q=0} \\
b_{k} & =a_{k}+J_{k} \geq 1
\end{aligned}
$$


We note from (3.20) that any variable $b_{k}$ is strictly larger than any variable $a_{k^{\prime}}$. The contribution of the graph $\gamma_{q}$ is

$$
\begin{aligned}
I_{\gamma_{q}}\left(J_{i}, K_{i}\right) & =(-)^{q} \delta_{J, K}\left(T_{X}-T_{Y}\right) \Phi_{\gamma_{q}}\left(n, J_{i}, K_{i}\right) \\
\Phi_{\gamma_{q}}\left(n, J_{i}, K_{i}\right) & =\sum_{j=0}^{n} \frac{h_{j+b_{1}} \ldots h_{j+b_{q}}}{h_{j+a_{1}} \ldots h_{j+a_{q}}}
\end{aligned}
$$

with

$$
\begin{aligned}
& 0 \leq X=\operatorname{Sup}\left(a_{k}\right) \\
& 1 \leq Y=\operatorname{Inf}\left(b_{k}\right)
\end{aligned}
$$

$\mathbf{2}^{\circ}$ ) the $\frac{1}{\xi}, \frac{1}{\eta}$ expansion for any graph: a one loop graph $\gamma$ is made of $q$ propagators $A, q$ propagators $K, q$ chains $\left(C_{1}, \ldots, C_{q}\right)$ of propagators $N$ and $q$ chains $\left(C_{1}^{*}, \ldots, C_{q}^{*}\right)$ of propagators $N^{*}$ (the chains can be reduced to a single point). The case $q=0$ is made of loops of $N$ propagators alone, or of $N^{*}$ propagators alone. To each chain $C_{i}$ we associate an external outgoing momentum

$$
\Delta_{i}=\sum_{\xi_{k} \in C_{i}} J_{k}
$$

and to each chain $C_{i}^{*}$ an external ingoing momentum

$$
\Theta_{i}=\sum_{\eta_{k} \in C_{i}^{*}} K_{k}
$$

Now, to each graph $\gamma$ we associate a skeleton graph $s(\gamma)$ obtained from $\gamma$ by shrinking into a point each of the chain $C_{i}$ or $C_{i}^{*}$. To each of these points we associate the corresponding external momentum $\Delta_{i}$ or $\Theta_{i}$; we have

$$
J=\sum_{i=1}^{q} \Delta_{i}=\sum_{i=1}^{q} \Theta_{i}=\sum_{\xi_{k} \in \gamma} J_{k}=\sum_{\eta_{k} \in \gamma} K_{k}=K
$$

Because all $J^{\prime} s$ and $K^{\prime} s$ are positive or nul, equation (3.26) shows that if the graph $\gamma$ is made of $N$ propagators alone $(q=0)$, since the $K^{\prime} s$ are zero, the $J^{\prime} s$ are also zero. The only non zero contribution in this category is the self-closed loop with one point which is $H_{n}\left(\xi_{i}, \xi_{i}\right)=-\frac{n+1}{\xi_{i}}$. The two points loop is

$$
" N\left(\xi_{i}, \xi_{j}\right) N\left(\xi_{j}, \xi_{i}\right) "=0
$$

Larger loops of $N$ propagators alone, or of $N^{*}$ propagators alone are shown in appendix A to be zero.

The functions $h_{i}$ are only present in the $A$ and $K$ propagators so that the function to be summed for a graph $\gamma$ is the characteristic function of the skeleton graph $s(\gamma)$

$$
\frac{h_{j+b_{1}} \ldots h_{j+b_{q}}}{h_{j+a_{1}} \ldots h_{j+a_{q}}}
$$


where $j$ is the momentum corresponding to the propagator $K_{q}$ and

$$
\begin{aligned}
a_{k} & =\sum_{p=1}^{k}\left(\Theta_{p}-\Delta_{p}\right), \quad a_{q=0} \\
b_{k} & =a_{k}+\Delta_{k} \geq 1
\end{aligned}
$$

The equation (3.10) for a one loop graph $\gamma$ with propagators $N$ and $N^{*}$ can be written

$$
I_{\gamma}\left(J_{i}, K_{i}\right)=(-)^{q} \delta_{J, K} O\left(J_{i}, K_{i}\right) \sum_{j=0}^{n} \frac{h_{j+b_{1}} \ldots h_{j+b_{q}}}{h_{j+a_{1}} \ldots h_{j+a_{q}}}
$$

where the operator $O\left(J_{i}, K_{i}\right)$ determins the range of summation for the variable $j$ and is described below.

We introduce the commutative operation $\otimes$

$$
\begin{aligned}
T_{a} \otimes T_{b} & =T_{\operatorname{Sup}(a, b)} \\
1 \otimes T_{b} & =T_{\operatorname{Sup}(0, b)}
\end{aligned}
$$

so that conditions like $n-a+1 \leq j \leq n$ together with $n-b+1 \leq j \leq n$, where $a$ and $b$ are positive, can be written

$$
\sum_{j=n-\operatorname{Inf}(a, b)+1}^{n} \ldots=\left(1-T_{a}\right) \otimes\left(1-T_{b}\right) \sum_{j=0}^{n} \ldots
$$

Now, we proved in appendix A that for a given chain $C_{i}$ with $p$ points $((p-1)$ propagators), the sum over the $p$ ! permutations generates a domain of summation for the variable $j_{i}$ described by the operator $\prod_{\xi_{r} \in C_{i}}\left(1-T_{J_{r}}\right)$; since the propagator with momentum $j_{i}$ is between the chains $C_{i}$ and $C_{i+1}^{*}\left(\right.$ with $C_{q+1}^{*}=C_{1}^{*}$ ) the constraints on $j_{i}$ can be written

$$
\left[\prod_{\xi_{r} \in C_{i}}\left(1-T_{J_{r}}\right)\right] \otimes\left[\prod_{\eta_{s} \in C_{i+1}^{*}}\left(1-T_{K_{s}}\right)\right]
$$

and using the relation (3.20) we can write the corresponding constraints on $j_{q}$ as

$$
T_{a_{i}}\left\{\left[\prod_{\xi_{r} \in C_{i}}\left(1-T_{J_{r}}\right)\right] \otimes\left[\prod_{\eta_{s} \in C_{i+1}^{*}}\left(1-T_{K_{s}}\right)\right]\right\}
$$

We collect all the constraints on the various momenta $j_{i}$ for $i=1, \ldots, q$ and write

$$
O\left(J_{i}, K_{i}\right)=\otimes_{i=1}^{q}\left[T_{a_{i}}\left\{\left[\prod_{\xi_{r} \in C_{i}}\left(1-T_{J_{r}}\right)\right] \otimes\left[\prod_{\eta_{s} \in C_{i+1}^{*}}\left(1-T_{K_{s}}\right)\right]\right\}\right]
$$




\section{The Gaussian potential, large $N$ and BMN expansion}

We now apply the results of section 3 to the Gaussian potential with the function $h_{n}$ given in (3.3); we also replace the index $n$ of section 3 by $(N-1)$ according to $(1.25)$.

$\left.1^{\circ}\right)$ let us consider the two points function

$$
W_{1,1}(N, x, y)=<\operatorname{Tr} \frac{1}{x-M} \operatorname{Tr} \frac{1}{y-M^{+}}>
$$

which is equal from (1.26) to the determinant

$$
" D_{N-1} "=" \operatorname{det} "\left|\begin{array}{ll}
H_{N-1}(x, x) & A_{N-1}(y, x) \\
K_{N-1}(x, y) & H_{N-1}(y, y)
\end{array}\right|
$$

The part $H_{N-1}(x, x) H_{N-1}(y, y)$ of the determinant corresponds to a disconnected graph made of two self closing loops and from (3.6.a) is equal to $\frac{N^{2}}{x y}$. The part $A_{N-1}(y, x) K_{N-1}(x, y)$ of the determinant corresponds to the graph $\gamma_{1}$ (fig.2). The corresponding function $\Phi_{\gamma_{1}}(N-1, J)$ is given in (3.14)

$$
\Phi_{\gamma_{1}}(N-1, J)=\sum_{j=0}^{N-1} \frac{\Gamma(j+J+1)}{\Gamma(j+1)}=\frac{1}{J+1} \frac{\Gamma(N+J+1)}{\Gamma(N)}
$$

Consequently, according to (3.15)

$A_{N-1}(y, x) K_{N-1}(x, y)=-\sum_{J=1}^{\infty} \frac{1}{(x y)^{J+1}} \frac{1}{J+1}\left[\frac{\Gamma(N+J+1)}{\Gamma(N)}-\frac{\Gamma(N+1)}{\Gamma(N-J)}\right]$

The square bracket [ ] in (4.4) has clearly a $\frac{1}{N^{2}}$ expansion

$$
\frac{\Gamma(N+J+1)}{\Gamma(N)}-\frac{\Gamma(N+1)}{\Gamma(N-J)}=2 N^{J} \sum_{p \text { odd }>0} \frac{1}{N^{p-1}} \sigma_{p}(J)
$$

where $\sigma_{p}(J)$ is described in appendix B. After a convenient rescaling, we write $A_{N-1}(y \sqrt{N}, x \sqrt{N}) K_{N-1}(x \sqrt{N}, y \sqrt{N})$ as

$$
\begin{aligned}
& -\frac{1}{N} \sum_{J=1}^{\infty} \frac{1}{(x y)^{J+1}}\left[J+\frac{1}{24 N^{2}}(J+1) J^{2}(J-1)(J-2)+O\left(\frac{1}{N^{4}}\right)\right] \\
& =-\frac{1}{N}\left[\frac{1}{(x y-1)^{2}}+\frac{1}{N^{2}} \frac{x y(3 x y+2)}{(x y-1)^{6}}+O\left(\frac{1}{N^{4}}\right)\right]
\end{aligned}
$$


The so-called BMN limit of $I_{\gamma_{1}}(J, K)$ (defined in (3.15), (4.3)) is the large $N$, large $J$ limit with constant $\frac{J^{2}}{N}, \frac{K^{2}}{N}$. From the large $J$ behaviour of $\sigma_{p}(J)$ as given in appendix B, we obtain the BMN limit as

$$
I_{\gamma_{1}}(J, K) \sim-\delta_{J, K} \frac{2 N^{J+1}}{J} \operatorname{sh}\left(\frac{J^{2}}{2 N}\right)
$$

At this stage, we find convenient to introduce a formalism developped in appendix B and which makes the $\frac{1}{N^{2}}$ expansion and the BMN limit transparant. We define

$$
N^{* *(J+1)}=\left(N-\frac{J}{2}\right)\left(N-\frac{J}{2}+1\right) \ldots\left(N+\frac{J}{2}-1\right)\left(N+\frac{J}{2}\right)
$$

which has manifestly a $\frac{1}{N^{2}}$ expansion

$$
N^{* *(J+1)}=\sum_{p=0}^{E\left(\frac{J+1}{2}\right)}(-)^{p} \Sigma_{2 p}(J) N^{J-2 p+1}
$$

where the coefficients $\Sigma_{2 p}(J)$ are given in appendix B. Consequently, the expression (4.4) can be written as

$$
\left(1-T_{J}\right) \frac{1}{J+1} \frac{\Gamma(N+J+1)}{\Gamma(N)}=\frac{2}{J+1} \operatorname{sh}\left(\frac{J}{2} \frac{\partial}{\partial N}\right) N^{* *(J+1)}
$$

which is manifestly a $\frac{1}{N^{2}}$ expansion. Also the BMN limit of $N^{* *(J+1)}$ is simply $N^{J+1}$ so that the BMN limit of (4.10) consists in replacing $\frac{\partial}{\partial N}$ by $\frac{J}{N}$ and $N^{* *(J+1)}$ by $N^{J+1}$.

$2^{\circ}$ ) we now consider the three points function

$$
W_{2,1}\left(N, x_{1}, x_{2}, y\right)=<\operatorname{Tr} \frac{1}{x_{1}-M} \operatorname{Tr} \frac{1}{x_{2}-M} \operatorname{Tr} \frac{1}{y-M^{+}}>
$$

which is equal from (1.26) to the determinant

$$
" D_{N-1} "=" \operatorname{det} "\left|\begin{array}{ccc}
H_{N-1}\left(x_{1}, x_{1}\right) & N_{N-1}\left(x_{2}, x_{1}\right) & A_{N-1}\left(y, x_{1}\right) \\
N_{N-1}\left(x_{1}, x_{2}\right) & H_{N-1}\left(x_{2}, x_{2}\right) & A_{N-1}\left(y, x_{2}\right) \\
K_{N-1}\left(x_{1}, y\right) & K_{N-1}\left(x_{2}, y\right) & H_{N-1}(y, y)
\end{array}\right|
$$

The connected contribution to (4.12) is

$$
\left[A_{N-1}\left(y, x_{1}\right) N_{N-1}\left(x_{1}, x_{2}\right) K_{N-1}\left(x_{2}, y\right)+\left(x_{1} \Leftrightarrow x_{2}\right)\right] .
$$




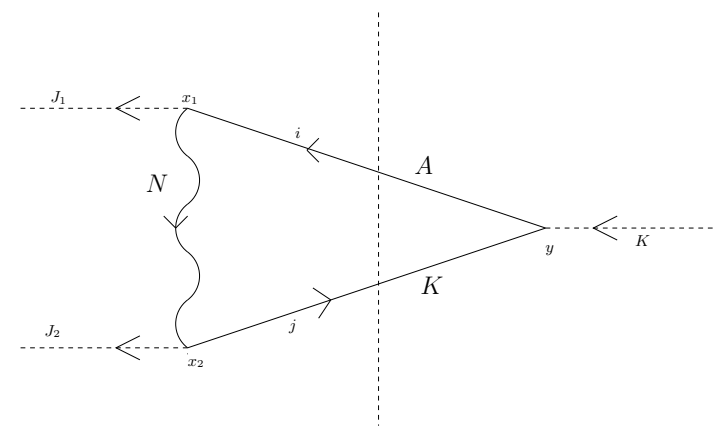

fig. 4 : contribution to $W_{2,1}^{c}\left(x_{1}, x_{2} ; y\right)$

In section 3 and in appendix $\mathrm{A}$, we prove that this combination is equal to

$$
\begin{aligned}
W_{2,1}^{c}\left(N, x_{1}, x_{2}, y\right) & =\sum_{\left\{J_{1}, J_{2}, K\right\}=1}^{\infty} \frac{1}{x_{1}^{J_{1}+1}} \frac{1}{x_{2}^{J_{2}+1}} \frac{1}{y^{K+1}} I_{2}\left(N, J_{1}, J_{2}, K\right) \\
I_{2}\left(N, J_{1}, J_{2}, K\right) & =-\delta_{J, K} \quad\left(1-T_{J_{1}}\right)\left(1-T_{J_{2}}\right) \frac{1}{J+1} \frac{\Gamma(N+J+1)}{\Gamma(N)} \\
I_{2}\left(N, J_{1}, J_{2}, K\right) & =-\delta_{J, K} \frac{4}{J+1} \operatorname{sh}\left(\frac{J_{1}}{2} \frac{\partial}{\partial N}\right) \operatorname{sh}\left(\frac{J_{2}}{2} \frac{\partial}{\partial N}\right) N^{* *(J+1)}
\end{aligned}
$$

where $J=K=J_{1}+J_{2}$. The large $N$ behaviour of

$W_{2,1}^{c}\left(N, x_{1} \sqrt{N}, x_{2} \sqrt{N}, y \sqrt{N}\right)$ is found to be

$$
\begin{aligned}
& -\frac{y}{N^{\frac{5}{2}}} \sum_{\left\{J_{1}, J_{2}\right\}=1}^{\infty} \frac{1}{\left(y x_{1}\right)^{J_{1}+1}} \frac{1}{\left(y x_{2}\right)^{J_{2}+1}} J_{1} J_{2} J * \\
& *\left[1+\frac{1}{24 N^{2}}(J-1)(J-2)\left\{(J+1)(J-2)-2 J_{1} J_{2}\right\}+O\left(\frac{1}{N^{4}}\right)\right]
\end{aligned}
$$

or

$$
W_{2,1}^{c}\left(N, x_{1} \sqrt{N}, x_{2} \sqrt{N}, y \sqrt{N}\right)=-\frac{2 y}{N^{\frac{5}{2}}}\left[\frac{y^{2} x_{1} x_{2}-1}{\left(y x_{1}-1\right)^{3}\left(y x_{2}-1\right)^{3}}+O\left(\frac{1}{N^{2}}\right)\right]
$$

Finally from (4.13.c), the BMN limit, where $N, J_{1}, J_{2}$ are large with

$\frac{J_{1}^{2}}{N}, \frac{J_{2}^{2}}{N}$ constant, is obtained as

$$
I_{2}\left(N, J_{1}, J_{2}, K\right) \sim-\delta_{J, K} \frac{4 N^{J+1}}{J} \operatorname{sh}\left(\frac{J_{1} J}{2 N}\right) \operatorname{sh}\left(\frac{J_{2} J}{2 N}\right)
$$


$3^{\circ}$ ) the generalisation of the situation $2^{\circ}$ ) is the calcumlation of

$W_{p, 1}^{c}\left(N, x_{1} \ldots, x_{p}, y\right)$ and is easy to understand. We sum over $p$ ! graphs obtained by exchanging the $p$ points $x_{i}$ and we calculate

$$
W_{p, 1}^{c}\left(N, x_{i}, y\right)=\left[A_{N-1}\left(y, x_{1}\right) \prod_{i=1}^{p-1} N_{N-1}\left(x_{i}, x_{i+1}\right) K_{N-1}\left(x_{p}, y\right)+\operatorname{Sym}\left(x_{i}\right)\right]
$$

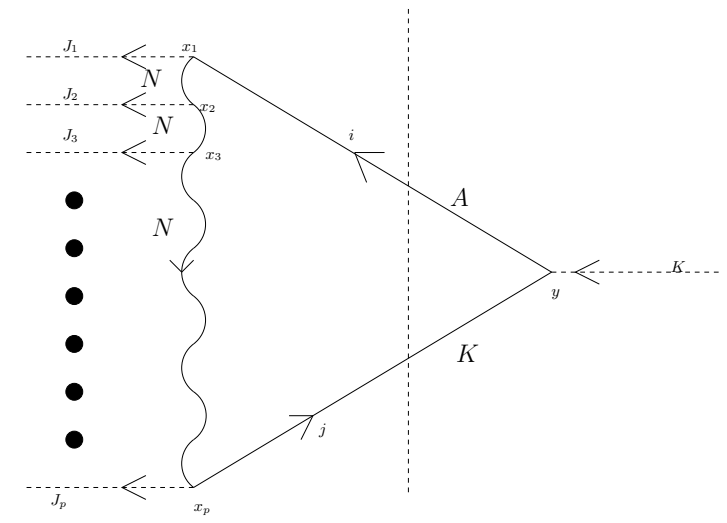

fig. 5 : contribution to $W_{p, 1}^{c}\left(x_{1}, \ldots, x_{p} ; y\right)$

From section 3 and appendix A, we know that the poles of the propagators $N_{N-1}\left(x_{i}, x_{i+1}\right)$ cancel in the sum and that the result can be written as

$$
\begin{aligned}
W_{p, 1}^{c}\left(N, x_{i}, y\right) & =\sum_{\left\{J_{i}, K\right\}=1}^{\infty} \prod_{i=1}^{p} \frac{1}{x_{i}^{J_{i}+1}} \frac{1}{y^{K+1}} I_{p}\left(N, J_{i}, K\right) \\
I_{p}\left(N, J_{i}, K\right) & =-\delta_{J, K} \prod_{i=1}^{p}\left(1-T_{J_{i}}\right) \frac{1}{J+1} \frac{\Gamma(N+J+1)}{\Gamma(N)} \\
I_{p}\left(N, J_{i}, K\right) & =-\delta_{J, K} \frac{2^{p}}{J+1} \prod_{i=1}^{p} \operatorname{sh}\left(\frac{J_{i}}{2} \frac{\partial}{\partial N}\right) N^{* *(J+1)}
\end{aligned}
$$

where $J=K=J_{1}+\ldots+J_{p}$. The asymptotic behaviour at large $N$ of

$W\left(N, x_{i} \sqrt{N}, y \sqrt{N}\right)$, after a proper rescaling of the variables, is

$-\frac{y^{p-1}}{N^{\frac{3 p-1}{2}}} \sum_{\left\{J_{i}\right\}=1}^{\infty} \prod_{i=1}^{p} \frac{1}{\left(x_{i} y\right)^{J_{i}+1}}\left[J_{1} \ldots J_{p} J(J-1) \ldots(J-p+2)+O\left(\frac{1}{N^{2}}\right)\right]$ 
or

$$
W_{p, 1}^{c}\left(N, x_{i} \sqrt{N}, y \sqrt{N}\right)=\frac{(-)^{p}}{N^{\frac{3 p-1}{2}}}\left\{\frac{\partial^{p-1}}{\partial y^{p-1}} \frac{y^{2 p-2}}{\prod_{i=1}^{p}\left(x_{i} y-1\right)^{2}}+O\left(\frac{1}{N^{2}}\right)\right\}
$$

The BMN limit is trivially obtained as

$$
I_{p}\left(N, J_{i}, K\right) \sim-\delta_{J, K} \frac{2^{p} N^{J+1}}{J} \prod_{i=1}^{p} s h\left(\frac{J_{i} J}{2 N}\right)
$$

$4^{\circ}$ ) We now apply the formalism to the four points, connected, resolvant function $W_{2,2}^{c}\left(N, x_{1}, x_{2}, y_{1}, y_{2}\right)$. First we calculate the contribution of the graph of fig.6 and its crossed symmetric ones $\left(J_{1} \Leftrightarrow J_{2}\right.$ and $\left.K_{1} \Leftrightarrow K_{2}\right)$.

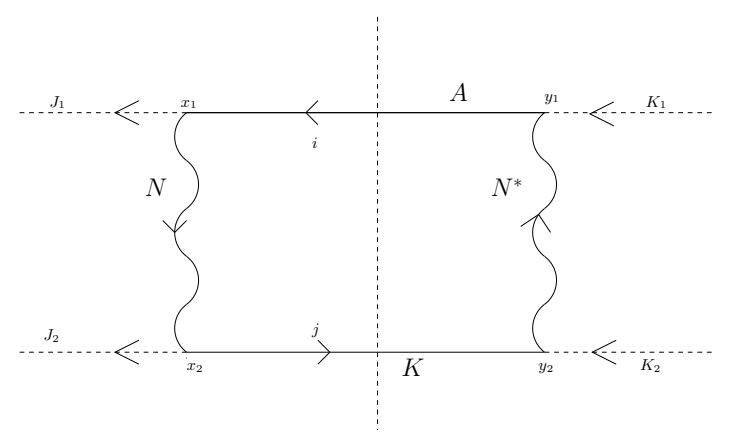

fig. 6 : contribution to $W_{2,2}^{c}\left(x_{1}, x_{2} ; y_{1}, y_{2}\right)$

We write

$$
W\left(N, x_{1}, x_{2}, y_{1}, y_{2}\right)=\sum_{\left\{J_{i}, K\right\}=1}^{\infty}\left(\prod_{i=1}^{2} \frac{1}{x_{i}^{J_{i}+1}}\right)\left(\prod_{i=1}^{2} \frac{1}{y_{i}^{K_{i}+1}}\right) I\left(N, J_{i}, K_{i}\right)
$$

The formalism of section 3 and appendix A applied to the corresponding four graphs gives

$$
\begin{aligned}
I\left(N, J_{i}, K_{i}\right) & =-\delta_{J, K} O\left(J_{i}, K_{i}\right) \frac{1}{J+1} \frac{\Gamma(N+J+1)}{\Gamma(N)} \\
O\left(J_{i}, K_{i}\right) & =\left[\left(1-T_{J_{1}}\right)\left(1-T_{J_{2}}\right) \otimes\left(1-T_{K_{1}}\right)\left(1-T_{K_{2}}\right)\right] \\
O\left(J_{i}, K_{i}\right) & =\left(1-T_{\text {Inf }}\right)\left(1+T_{\text {Sup }}\right)
\end{aligned}
$$

where the symbol $\otimes$ is defined in (3.31) and where

$$
\begin{aligned}
J & =K=J_{1}+J_{2}=K_{1}+K_{2} \\
\text { Inf } & =\operatorname{Inf}\left(J_{i}, K_{i}\right) \quad \operatorname{Sup}=\operatorname{Sup}\left(J_{i}, K_{i}\right)
\end{aligned}
$$


The function $I\left(N, J_{i}, K_{i}\right)$ can also be written

$$
I\left(N, J_{i}, K_{i}\right)=-\delta_{J, K} \frac{4}{J+1} \operatorname{sh}\left(\frac{\operatorname{Inf}}{2} \frac{\partial}{\partial N}\right) \operatorname{ch}\left(\frac{\operatorname{Sup}}{2} \frac{\partial}{\partial N}\right) N^{* *(J+1)}
$$

The BMN limit is immediately obtained as

$$
I\left(N, J_{i}, K_{i}\right) \sim-\delta_{J, K} \frac{4 N^{J+1}}{J} \operatorname{sh}\left(\frac{J . I n f}{2 N}\right) \operatorname{ch}\left(\frac{J . S u p}{2 N}\right)
$$

The large $N$ limit is obtained by expansion of the $s h$ and $c h$ in (4.25) as

$$
\begin{aligned}
I\left(N, J_{i}, K_{i}\right) & =-\delta_{J, K} 2 \operatorname{Inf} N^{J} * \\
& *\left[1+\frac{J(J-1)}{24 N^{2}}\left[3 \operatorname{Sup}^{2}+\operatorname{Inf} f^{2}-(J+2)\right]+O\left(\frac{1}{N^{4}}\right)\right]
\end{aligned}
$$

$5^{\circ}$ ) The remaining part of the connected four points resolvant function $W_{2,2}^{c}\left(N, x_{1}, x_{2}, y_{1}, y_{2}\right)$ is attached to the skeleton graphs of fig. 3 and its crossed symmetric one $\left(J_{1} \Leftrightarrow J_{2}\right)$ or $\left(K_{1} \Leftrightarrow K_{2}\right)$. According to section 3 , we have for the graph of fig. 3

$$
I_{1}\left(N, J_{i}, K_{i}\right)=\delta_{J, K}\left(T_{X}-T_{Y}\right) \sum_{j=0}^{N-1} \frac{\Gamma\left(j+K_{1}+1\right)}{\Gamma\left(j+K_{1}-J_{1}+1\right)} \frac{\Gamma\left(j+J_{2}+1\right)}{\Gamma(j+1)}
$$

with $J=J_{1}+J_{2}=K_{1}+K_{2}=K$ and with

$$
X=\operatorname{Sup}\left(K_{1}-J_{1}, 0\right) \quad Y=\operatorname{Inf}\left(K_{1}, J_{2}\right)
$$

The sum in (4.28) is transformed in appendix B (B.31) into

$$
\sum_{p=0}^{\operatorname{Inf}\left(J_{1}, K_{2}\right)}(-)^{p} p ! C_{J_{1}}^{p} C_{K_{2}}^{p} \frac{\Gamma(N+J-p+1)}{\Gamma(N)}
$$

We can now compute the large $N$ behaviour of $I_{1}\left(N, J_{i}, K_{i}\right)$; for instance, in the sector $J_{1} \geq K_{1}, K_{2} \geq J_{2}$ we obtain

$$
\begin{array}{r}
I_{1}\left(N, J_{i}, K_{i}\right)=\delta_{J, K} J_{2} N^{J}\left[1+\frac{J_{1}\left(K_{1}-K_{2}\right)}{2 N}+\frac{1}{24 N^{2}} \digamma+O\left(\frac{1}{N^{3}}\right)\right] \\
\digamma=J(J-1)\left[3 J_{1}^{2}+J_{2}^{2}-(J+2)\right]-12 K_{1} K_{2} J_{1}\left(J_{1}-1\right)
\end{array}
$$

For the BMN behaviour, each term in (4.30) contributes, however the sum of these contributions can be performed and we obtain

$$
I_{1}\left(N, J_{i}, K_{i}\right) \sim \delta_{J, K} \frac{2 N^{J+1}}{J} \operatorname{sh}\left(\frac{J_{2} J}{2 N}\right) e^{\frac{J_{1}\left(K_{1}-K_{2}\right)}{2 N}}
$$


If we sum the graph of fig. 3 and the crossed one, and if we describe each of the sectors as

$$
\text { Sup } \geq A, B \geq \operatorname{Inf}
$$

with $A+B=S u p+I n f=J=K$, we obtain the large $N$ behaviour for both graphs as

$$
\begin{aligned}
I\left(N, J_{i}, K_{i}\right) & =\delta_{J, K} 2 \operatorname{Inf} N^{J}\left[1+\frac{1}{24 N^{2}} \digamma+O\left(\frac{1}{N^{4}}\right)\right] \\
\digamma & =J(J-1)\left[3 \operatorname{Sup}^{2}+I_{n} f^{2}-(J+2)\right]-12 \text { AB Sup }(\text { Sup }-1)
\end{aligned}
$$

and the BMN behaviour as

$$
I\left(N, J_{i}, K_{i}\right) \sim \delta_{J, K} \frac{4 N^{J+1}}{J} \operatorname{sh}\left(\frac{J . I n f}{2 N}\right) \operatorname{ch}\left(\frac{(A-B) \cdot S u p}{2 N}\right)
$$

$6^{\circ}$ ) Finally, we sum the contributions $4^{\circ}$ ) and $5^{\circ}$ ) to obtain the asymptotic behaviour for the complete connected four points correlation function; taking into account the relative minus sign between $4^{\circ}$ ) and $5^{\circ}$ ), we obtain

$$
\begin{aligned}
& \Gamma_{2}^{c}\left(N, J_{i}, K_{i}\right)=-\delta_{J, K} \quad N^{J-2} \quad J_{1} J_{2} K_{1} K_{2}(\text { Sup }-1)+O\left(N^{J-4}\right) \\
& \Gamma_{2}^{c}\left(N, J_{i}, K_{i}\right) \sim-\delta_{J, K} \frac{8 N^{J+1}}{J} \operatorname{sh}\left(\frac{\text { J.Inf }}{2 N}\right) \operatorname{sh}\left(\frac{\text { A.Sup }}{2 N}\right) \operatorname{sh}\left(\frac{\text { B.Sup }}{2 N}\right)
\end{aligned}
$$

We close this section with the following remarks. Although, in principle, we should be able to calculate the contribution of any graph, we are unable at the present time to give the expression for the sum of the graphs which enters a given $k$-points correlation function; this situation may improve if we use the so-called loop equations[12]. From the exemple $5^{\circ}$ ), we see that the t'Hooft topological expansion in $\frac{1}{N^{2}}$ is not true graph by graph and that the cancellations between graphs are far from evident. Finally, we verify on these exemples that at large $N$ the resolvant function properly rescaled behave as

$$
W_{k_{1}, k_{2}}\left(N, x_{i} \sqrt{N}, y_{i} \sqrt{N}\right) \rightarrow O\left(N^{\frac{k}{2}}\right)
$$

because of the disconnected one point self closing loops (the lowest term of the expansion) and that the connected resolvant function properly rescaled behave as

$$
W_{k_{1}, k_{2}}^{c}\left(N, x_{i} \sqrt{N}, y_{i} \sqrt{N}\right) \rightarrow O\left(\frac{1}{N^{\frac{3 k-4}{2}}}\right)
$$

where $k=k_{1}+k_{2}$. 


\section{A Appendix: summation over the chains of prop- agators $N$ and $N^{*}$}

We consider a chain of $(p-1)$ propagators $N$ (the arguments are the same for the chains of propagators $\left.N^{*}\right)$. The propagator $N_{n}\left(\xi_{i}, \xi_{i+1}\right)$ has a pole at $\xi_{i}=\xi_{i+1}$. However, when we sum over the $p$ ! chains obtained by exchanging the $p$ points, the poles disappear as we explain now: we consider two points $\xi_{b}$ and $\xi_{c}$ and we look for the pole at $\xi_{b}=\xi_{c}$; many chains do not contain the propagators $N_{n}\left(\xi_{b}, \xi_{c}\right)$ or $N_{n}\left(\xi_{c}, \xi_{b}\right)$ and have no pole at $\xi_{b}=\xi_{c}$. Now, we associate by pair the chains with a propagator $N_{n}\left(\xi_{b}, \xi_{c}\right)$ or $N_{n}\left(\xi_{c}, \xi_{b}\right)$ by exchanging $b$ and $c$; the combination

$\left(N_{n}\left(\xi_{a}, \xi_{b}\right) N_{n}\left(\xi_{b}, \xi_{c}\right) N_{n}\left(\xi_{c}, \xi_{d}\right)+N_{n}\left(\xi_{a}, \xi_{c}\right) N_{n}\left(\xi_{c}, \xi_{b}\right) N_{n}\left(\xi_{b}, \xi_{d}\right)\right)$ has no pole at $\xi_{b}=\xi_{c}$ and is equal to

$$
\ldots\left(\frac{\xi_{a}}{\xi_{d}}\right)^{n+1} \frac{\xi_{d}-\xi_{a}}{\left(\xi_{b}-\xi_{a}\right)\left(\xi_{d}-\xi_{c}\right)\left(\xi_{c}-\xi_{a}\right)\left(\xi_{d}-\xi_{b}\right)} \cdots
$$

Similar arguments prove the absence of poles at $\xi_{b}=\xi_{c}$ if $b$ or/and $c$ are endpoints of the chain, for instance in $\left(N_{n}\left(\xi_{b}, \xi_{c}\right)+N_{n}\left(\xi_{c}, \xi_{b}\right)\right)$ or in $\left(N_{n}\left(\xi_{a}, \xi_{b}\right) N_{n}\left(\xi_{b}, \xi_{c}\right)+N_{n}\left(\xi_{a}, \xi_{c}\right) N_{n}\left(\xi_{c}, \xi_{b}\right)\right)$. We remind that the self closing loops $N_{n}\left(\xi_{b}, \xi_{b}\right)$ are replaced in (1.26) by $H_{n}\left(\xi_{b}, \xi_{b}\right)$ given in (3.6.a) and contribute only to disconnected parts of the graphs $\left(J_{i}=0\right)$ and that the two points loop $N_{n}\left(\xi_{b}, \xi_{c}\right) N_{n}\left(\xi_{c}, \xi_{b}\right)$ is replaced in (1.26) by " $N_{n}\left(\xi_{b}, \xi_{c}\right) N_{n}\left(\xi_{c}, \xi_{b}\right)$ " which has no pole and is zero in rotationnally invariant systems.

Consequently, since we have no poles at coinciding $\xi^{\prime} s$, the expansion in $\frac{1}{\xi_{i}}$ for the contribution of the $p$ ! chains is the same whatever sector we choose $\xi_{\alpha_{1}} \leq \xi_{\alpha_{2}} \leq \ldots \leq \xi_{\alpha_{p}}$ and the results must contain terms of the type $\left(\frac{1}{\xi_{i}}\right)^{J_{i}+1}$ with $J_{i}>0$ if $p>1$. According to the chosen sector we use the convergent expansion

$$
\begin{aligned}
& \frac{1}{\xi_{i+1}-\xi_{i}}=\frac{1}{\xi_{i+1}} \sum_{j=0}^{\infty}\left(\frac{\xi_{i}}{\xi_{i+1}}\right)^{j}, \quad \xi_{i}<\xi_{i+1} \\
& \frac{1}{\xi_{i+1}-\xi_{i}}=-\frac{1}{\xi_{i}} \sum_{j=0}^{\infty}\left(\frac{\xi_{i+1}}{\xi_{i}}\right)^{j}, \quad \xi_{i}>\xi_{i+1}
\end{aligned}
$$

Now, each chain separetely can be expanded in the choosen sector but if we find in the expansion some contributions $\left(\frac{1}{\xi_{i}}\right)^{J_{i}+1}$ with a negative or nul power $J_{i}$, we may ignore that contribution since we know that it must be cancelled when we sum over the $p$ ! chains.

For instance, we consider three consecutive points of the chain and the corresponding situation

$$
N_{n}\left(\xi_{i-1}, \xi_{i}\right) N_{n}\left(\xi_{i}, \xi_{i+1}\right), \quad \xi_{i}=\operatorname{Inf}\left(\xi_{i-1}, \xi_{i}, \xi_{i+1}\right)
$$


according to (A.2) we obtain the contribution

$$
-\frac{1}{\xi_{i-1} \xi_{i+1}}\left(\frac{\xi_{i-1}}{\xi_{i+1}}\right)^{n+1} \sum_{r, s=0}^{\infty}\left(\frac{\xi_{i}}{\xi_{i-1}}\right)^{r}\left(\frac{\xi_{i}}{\xi_{i+1}}\right)^{s}
$$

This term provides for the variable $\xi_{i}$ a contribution

$$
\left(\frac{1}{\xi_{i}}\right)^{-r-s} \rightarrow J_{i}=-r-s-1<0
$$

so that this chain should be ignored in the choosen sector. In such a sector, only the chains with no other relative minimum that the end-points of the chain should be considered.

A first important consequence of this remark is that the chains which form a loop gives no contribution; this can be verified for instance in (A.1) with $a=d$

in the case of a three points loop. A second consequence is that if we compute

$$
-A_{n}\left(\eta_{1}^{*}, \xi_{b_{1}}\right) \prod_{i=1}^{p-1} N_{n}\left(\xi_{b_{i}}, \xi_{b_{i+1}}\right) K_{n}\left(\xi_{b_{p}}, \eta_{2}^{*}\right)
$$

in the sector $\xi_{\alpha_{1}} \leq \xi_{\alpha_{2}} \leq \ldots \leq \xi_{\alpha_{p}}$ then, $\xi_{\alpha_{1}}$ is either $\xi_{b_{1}}$ or $\xi_{b_{p}}$ and along the chain we have

$$
\xi_{b_{1}} \leq \xi_{b_{2}} \leq \ldots \leq \xi_{b_{q}}=\xi_{\alpha_{p}} \geq \xi_{b_{q+1}} \geq \xi_{b_{q+2}} \geq \ldots \geq \xi_{b_{p}} \quad 1 \leq q \leq p
$$

From (A.2), we obtain for (A.6)

$$
\begin{aligned}
& (-)^{p-q} \frac{1}{\left(\eta_{1}^{*}\right)^{i+1}}\left(\frac{1}{\xi_{b_{1}}}\right)^{i-n-r_{1}} \prod_{i=2}^{q-1}\left(\frac{1}{\xi_{b_{i}}}\right)^{r_{i-1}-r_{i}+1} * \\
& *\left(\frac{1}{\xi_{b_{q}}}\right)^{r_{q-1}+r_{q}+2} \prod_{i=q+1}^{p-1}\left(\frac{1}{\xi_{b_{i}}}\right)^{r_{i}-r_{i-1}+1}\left(\frac{1}{\xi_{b_{p}}}\right)^{n-j-r_{p-1}+1} \frac{1}{\left(\eta_{2}^{*}\right)^{-j}}
\end{aligned}
$$

and by identification, we have

$$
\begin{array}{ll}
J_{b_{1}}=i-n-r_{1}-1 & \\
J_{b_{i}}=r_{i-1}-r_{i} & 2 \leq i \leq q-1 \\
J_{b_{q}}=r_{q-1}+r_{q}+1 & \\
J_{b_{i}}=r_{i}-r_{i-1} & q+1 \leq i \leq p-1 \\
J_{b_{p}}=n-j-r_{p-1} &
\end{array}
$$

which implies

$$
J=\sum_{i=1}^{p} J_{b_{i}}=i-j
$$


Clearly, the variables $i$ and $r_{i}$ are all determined as function of $n, j$ and $J_{b_{i}}$; the constraints $i \geq n+1$ and $r_{i} \geq 0$ together with $J_{b_{i}}>0$ implies only two constraints on $j$

$$
\begin{aligned}
& j \leq n-J_{+} \\
& j \geq n-J_{+}-J_{b_{q}}+1
\end{aligned}
$$

with

$$
J_{+}=\sum_{i=q+1}^{p} J_{b_{i}}
$$

The original sum over $j$ of the function $\frac{h_{i}}{h_{j}}$ describing the kernels $A_{n}\left(\eta_{1}^{*}, \xi_{b_{1}}\right)$ and $K_{n}\left(\xi_{b_{p}}, \eta_{2}^{*}\right)$ is, for that chain, transformed into

$$
\sum_{j=0}^{n} \frac{h_{j+J}}{h_{j}} \ldots \Rightarrow(-)^{p-q} \sum_{j=n-J_{+}-J_{b_{q}}+1}^{n-J_{+}} \frac{h_{j+J}}{h_{j}}=(-)^{p-q} T_{J_{+}}\left(1-T_{J_{a_{p}}}\right) \sum_{j=0}^{n} \frac{h_{j+J}}{h_{j}} \ldots
$$

where the operator $T_{a}$ is defined in (3.16).

Finally, summing over all the $p$ ! chains consists in summing over all subsets

of $\left\{\alpha_{1}, \ldots, \alpha_{p-1}\right\}$ (including the empty set and the complete set) corresponding to the definition of $J_{+}$. Taking into account the alternate sign in $(-)^{p-q}$ the total sum is nothing but

$$
\prod_{i=1}^{p}\left(1-T_{J_{i}}\right) \sum_{j=0}^{n} \frac{h_{j+J}}{h_{j}} \ldots
$$

This result is the purpose of this appendix.

\section{B Appendix: the 't Hooft expansion}

We define the symbol

$$
n^{* J}=(n+1)(n+2) \ldots(n+J)
$$

which is expanded in powers of $n$ as

$$
n^{* J}=\sum_{p=0}^{J} \sigma_{p}(J) n^{J-p}
$$

with

$$
\begin{array}{lrl}
\sigma_{0}(J) & =1 & \sigma_{J}(J)=J ! \\
\sigma_{1}(J)=\frac{J(J+1)}{2} & \sigma_{p}(J)=0 & \text { if } p>J
\end{array}
$$


For a given $p$, the coefficients $\sigma_{p}(J)$ are polynomials in $J$; clearly we have

$$
\sigma_{p}(J)=\sum_{1 \leq k_{1}<k_{2}<\ldots<k_{p} \leq J} k_{1} k_{2} \ldots k_{p}
$$

and we observe the relation

$$
\sigma_{p}(J)=\sigma_{p}(J-1)+J \sigma_{p-1}(J-1)
$$

Since $\sigma_{p}(J)$ vanishes if $J<p$, we write

$$
\begin{array}{rrr}
\sigma_{p}(J) & =\frac{1}{2^{p} p !} J(J-1) \ldots(J-p+1) \pi_{p}(J) \\
\pi_{0}(J) & =1 & \pi_{1}(J)=J+1
\end{array}
$$

The relation

$$
J \pi_{p}(J)=(J-p) \pi_{p}(J-1)+2 p J \pi_{p-1}(J-1)
$$

shows that $\pi_{p}(J)$ is a monic polynomial of degree $p$ in $J$ which vanishes at $J=-1$ if $p>0$.We find

$$
\begin{aligned}
& \pi_{2}(J)=(J+1)\left(J+\frac{2}{3}\right) \quad \pi_{3}(J)=J(J+1)^{2} \\
& \pi_{4}(J)=(J+1)\left(J^{3}+J^{2}-\frac{2}{3} J-\frac{8}{15}\right) \\
& \pi_{5}(J)=J(J+1)^{2}\left(J^{2}-\frac{1}{3} J-2\right)
\end{aligned}
$$

We note that at large $J$

$$
\sigma_{p}(J) \sim \frac{1}{p !}\left(\frac{J^{2}}{2}\right)^{p}
$$

so that the so called "BMN" limit ( $n$ and $J \rightarrow \infty, \frac{J^{2}}{n}=$ cte) is

$$
n^{* J} \sim n^{J} e^{\frac{J^{2}}{2 n}}
$$

From the generating functional

$$
(1+x)^{-j-1}=\sum_{J=0}^{\infty}(-)^{J} j^{* J} \frac{x^{J}}{J !}
$$

and from

$$
\sum_{j=0}^{n}(1+x)^{-j-1}=\frac{1-(1+x)^{-n-1}}{x}
$$


we obtain by identification of the powers of $x$

$$
\sum_{j=0}^{n} j^{* J}=\frac{n^{*(J+1)}}{J+1}
$$

In section 4 we have to calculate

$$
\sum_{j=N-J}^{N-1} j^{* J}=\frac{(N-1)^{*(J+1)}-(N-J-1)^{*(J+1)}}{J+1}
$$

From

$$
\begin{gathered}
(N-1)^{*(J+1)}=N(N+1) \ldots(N+J)=\sum_{p=0}^{J} \sigma_{p}(J) N^{J-p+1} \\
(N-J-1)^{*(J+1)}=N(N-1) \ldots(N-J)=\sum_{p=0}^{J}(-)^{p} \sigma_{p}(J) N^{J-p+1}
\end{gathered}
$$

we obtain the topological t'Hooft expansion

$$
\sum_{j=N-J}^{N-1} j^{* J}=\frac{2}{J+1} \sum_{p \text { odd }}^{J} \sigma_{p}(J) N^{J-p+1}
$$

Also, from (B.9), the BMN limit (large $N$, large $J, \frac{J^{2}}{N}=c t e$ ) is

$$
\sum_{j=N-J}^{N-1} j^{* J} \sim \frac{2 N^{J+1}}{J} \operatorname{sh}\left(\frac{J^{2}}{2 N}\right)
$$

We find convenient to introduce the expression

$$
\begin{aligned}
& n^{* *(J+1)}=e^{-\left(\frac{J}{2}+1\right) \frac{\partial}{\partial n}} n^{*(J+1)} \\
& n^{* *(J+1)}=\left(n-\frac{J}{2}\right)\left(n-\frac{J}{2}+1\right) \ldots\left(n+\frac{J}{2}-1\right)\left(n+\frac{J}{2}\right)
\end{aligned}
$$

which has clearly a $\frac{1}{n^{2}}$ expansion

$$
n^{* *(J+1)}=\sum_{p=0}^{E\left(\frac{J+1}{2}\right)}(-)^{p} \Sigma_{2 p}(J) n^{J-2 p+1}
$$


with

$$
\Sigma_{0}(J)=1 \quad \Sigma_{2}(J)=\frac{J(J+1)(J+2)}{24}
$$

We have the relation

$$
\Sigma_{2 p}(J)=\Sigma_{2 p}(J-2)+\frac{J^{2}}{4} \Sigma_{2 p-2}(J-2)
$$

We write for $p>0$

$$
\begin{aligned}
\Sigma_{2 p}(J) & =\frac{(J-2 p+2)(J-2 p+3) \ldots J(J+1)}{(24)^{p}} \quad \theta_{2 p}(J) \\
\theta_{0}(J) & =1 \quad \theta_{2}(J)=J+2
\end{aligned}
$$

the relation (B.21) becomes

$$
J(J+1) \quad \theta_{2 p}(J)=(J-2 p)(J-2 p+1) \theta_{2 p}(J-2)+6 p J^{2} \theta_{2 p-2}(J-2)
$$

The polynomials $\theta_{2 p}(J)$ are monic polynomials of degree $p$ in $J$ which vanish for $J=-2$ if $p>0$. We have

$$
\begin{aligned}
& \theta_{4}(J)=(J+2)\left(J+\frac{12}{5}\right) \\
& \theta_{6}(J)=(J+2)\left(J^{2}+\frac{26}{5} J+\frac{48}{7}\right) \\
& \theta_{8}(J)=(J+2)\left(J^{3}+\frac{42}{5} J^{2}+\frac{4184}{175} J+\frac{576}{25}\right)
\end{aligned}
$$

At large $J$

$$
\Sigma_{2 p}(J) \sim \frac{1}{p !}\left(\frac{J^{3}}{24}\right)^{p}
$$

so that the "BMN" limit (and the first correction) of $n^{* *(J+1)}$ is

$$
n^{* *(J+1)} \sim n^{J+1} \quad e^{-\frac{J^{3}}{24 n^{2}}}=n^{J+1}\left[1+O\left(\frac{1}{J}\right)\right], \quad \frac{J^{2}}{n}=c t e
$$

the exponential term being the first correction to the "BMN" limit.

With these notations, we may compute directly the $\frac{1}{N^{2}}$ expansion of

$$
\prod_{i=1}^{p}\left(1-T_{J_{i}}\right) \sum_{k=0}^{N-1} \frac{\Gamma(k+J+1)}{\Gamma(k+1)}=\frac{2^{p}}{J+1} \prod_{i=1}^{p}\left[s h\left(\frac{J_{i}}{2} \frac{\partial}{\partial N}\right)\right] N^{* *(J+1)}
$$

where $\sum_{i=1}^{p} J_{i}=J$; the "BMN" limit is trivially

$$
\prod_{i=1}^{p}\left(1-T_{J_{i}}\right) \sum_{k=0}^{N-1} \frac{\Gamma(k+J+1)}{\Gamma(k+1)} \sim 2^{p} \frac{N^{J+1}}{J} \prod_{i=1}^{p}\left[\operatorname{sh}\left(\frac{J_{i} J}{2 N}\right)\right] \quad, \quad \frac{J_{i} J}{N}=c t e
$$


Finally, if we compare the coefficients of the term $x^{b}$ in the expansions

$$
(1+x)^{-j-1}=(1+x)^{-j-a-1}(1+x)^{a}
$$

we obtain the relation

$$
\frac{\Gamma(j+b+1)}{\Gamma(j+1)}=\sum_{q=0}^{\operatorname{Inf}(a, b)}(-)^{q} q ! C_{a}^{q} C_{b}^{q} \frac{\Gamma(j+a+b-q+1)}{\Gamma(j+a+1)}
$$

if $a$ is a non negative integer (with trivial analytic continuation to any $a$ ). As a consequence

$$
\begin{aligned}
& \sum_{j=0}^{N-1} \frac{\Gamma(j+a+c+1)}{\Gamma(j+1)} \frac{\Gamma(j+b+c+1)}{\Gamma(j+c+1)} \\
& =\sum_{q=0}^{\operatorname{Inf}(a, b)}(-)^{q} \frac{q ! C_{a}^{q} C_{b}^{q}}{(a+b+c-q+1)} \frac{\Gamma(N+a+b+c-q+1)}{\Gamma(N)}
\end{aligned}
$$

Acknowledgements: I wish to thank B. Eynard and C.F. Kristjansen who suggested this calculation.

\section{References}

[1] Bergère M.C.: "biorthogonal polynomials for potentials of two variables and external sources at the denominator", hep-th/0404126

[2] Szegö G: orthogonal polynomials, Am. Math. Soc. Colloquium publications 23, Providence (1975)

[3] Uvarov V.B.: USSR Comput. Math. 9, $\mathrm{n}^{\circ}$ ) 6, 25-36 (1969)

[4] Fyodorov Y. and Strahov E.: J. of Physics A36, 3203-3213 (2003)

[5] Akemann G. and Vernizzi G.: Nucl. Phys. B660, 532-556 (2003)

[6] Bergère M.C.: "orthogonal polynomials for potentials of two variables with external sources", hep-th/0311227

[7] Akemann G. and Pottier A.: J.Phys.A37, L453-L460 (2004)

[8] t'Hooft G.: Nucl. Phys. B72, 461-473 (1974)

[9] Berenstein D., Maldacena J. and Nastase H.: JHEP 0204, 013 (2002 
[10] Beisert N., Kristjansen C., Plefka J., Semenoff G.W. and Staudacher M.: Nucl. Phys. B650, 125-161 (2003)

[11] Bergère M.C.: 4th Eurogrid meeting, Les Houches (march 2004); Capri meeting, "From Bohr atomic scale to Planck scale", Sept 20 (2004)

[12] Eynard B. and Orantin N.: "A residue formula for correlation functions for the hermitian two matrix model", math-ph/0504058 